\title{
Caloric Restriction Affects Liver Microsomal Monooxygenases Differentially in Aging Male Rats
}

\author{
Douglas L. Schmucker,' Rose K. Wang, 'David Snyder, ${ }^{2}$ \\ Henry Strobel, ${ }^{3}$ and Ulrich Marti ${ }^{4}$
}

\author{
'Cell Biology and Aging Section, VA Medical Center, and the University of California, San Francisco. \\ ${ }^{2}$ Lobund Laboratory, University of Notre Dame. \\ ${ }^{3}$ University of Texas Health Science Center, Houston. \\ ${ }^{4}$ Department of Clinical Pharmacology, University of Berne, Switzerland.
}

\begin{abstract}
Caloric restriction (CR) extends life span and retards the onset of physiological changes and pathologies associated with aging, but the underlying mechanisms remain unresolved. This study demonstrates that $C R$ postpones the documented age-related declines in andlor enhances the activity and microsomal concentrations of several liver monooxygenases in male rats, i.e., NADPH cytochrome $P-450$ reductase, total cytochromes $P-450$. However, the relative concentration of cytochrome $P-450_{b+e}$ did not exhibit statistically significant changes, whereas another isozyme, the male specific $P-450_{\mathrm{h}}$, declined significantly in both ad libitum-fed and CR rats as a function of increasing age. While CR appears to retard age-associated changes in certain liver enzymes, this effect is by no means universal. The hepatic monooxygenases constitute a well-characterized enzyme system in which to examine the perturbation of the aging process by $C R$.
\end{abstract}

$\mathrm{C}$ ALORIC restriction significantly and nearly universally extends life span (see Masoro, 1985, for a review). Reducing caloric intake retards age-related declines in many physiological processes and postpones the onset of ageassociated pathologies (see Masoro, 1985; Walford et al., 1987 , for reviews). However, the mechanism whereby dietary restriction effects these changes remains unresolved. Recently, Richardson et al. (1987) demonstrated that age and dietary restriction-induced changes in the expression of a specific hepatic protein, $\alpha_{24}$-globulin, are elicited at the level of transcription. Unfortunately, the facts that (a) $\alpha_{2 u^{-}}$ globulin synthesis is enhanced by androgens, (b) old male Fischer 344 rats, which are characterized by a high incidence of testicular interstitial cell tumors $(90-100 \%)$, were used, and (c) the function of this protein remains unknown, temper the interpretation of these data. Furthermore, this same laboratory has been unable to demonstrate similar age and/or dietary-induced alterations in the urinary excretion of $\alpha_{2 u^{-}}$ globulin in male Lobund-Wistar rats (Wu et al., 1989). Comparable studies on well-characterized proteins and enzymes are required to resolve the mechanism whereby dietary restriction influences the aging process.

The hepatic microsomal monooxygenases are well-characterized, and the effects of aging on this system have been subjected to extensive study, at least in inbred male rats (see Schmucker, 1985, for a review). Aging impairs certain constituents of this drug-metabolizing pathway in male rats, and this impairment is expressed as (a) reduced levels and activities of monooxygenases (Schmucker and Wang, 1980a; 1980b), (b) impaired phenobarbital-induction of monooxygenases (Schmucker and Wang, 1981), and (c) the accumulation of catalytically inactive NADPH cytochrome P-450 reductase (Schmucker and Wang, 1983). Other investigators reported marked age and sex-dependent shifts in the profile of cytochrome P-450 isozymes in male rats, i.e., a "feminization" of the male monooxygenase system (Kamataki et al., 1985; Kitagawa et al., 1985). Hepatic microsomal monooxygenases constitute a suitable model in which to study the mechanism(s) whereby caloric restriction perturbs the aging process. This report describes our initial studies on the effects of caloric restriction on hepatic microsomal monooxygenases of male Lobund-Wistar rats as a function of aging.

\section{METHODS}

Animals. - Ad libitum or full-fed (FF) and caloricrestricted $(\mathrm{CR})$ male Lobund-Wistar $(\mathrm{L}-\mathrm{W})$ rats were segregated into age groups of 7 months (young adults), 18 months (mature), 26-36 months (old), and 37-42 months (very old, CR only). The animals, part of the Lobund Aging Study, were maintained in the animal care facility of the Lobund Laboratory, University of Notre Dame. They were pathogen-free, exhibited mild nephropathy only after 30 months, and expressed median survival of 31 and 39 months and maximum life spans of 38.9 and 50.9 months in the FF and CR groups, respectively (Snyder, 1989). The maintenance conditions and feeding regimens were identical to those described by Snyder and Wostmann (1987). The latter consisted of feeding a sterile, natural ingredient diet (L-485; $20 \%$ protein, $5 \%$ fat, $3 \%$ fiber) either ad libitum or restricted (12 gm per day; 30\% restriction) (Snyder et al., 1990). The restricted diet was initiated at 6 weeks of age and animals under this regimen never received more than $12 \mathrm{~g}$ of food per day for the remainder of their life span.

Analyses. - Animals from each age and diet group were killed between 0900 and $1100 \mathrm{hrs}$ following an overnight 
fast ( $16 \mathrm{hrs}$ ). The livers were immediately removed, flashfrozen in liquid $\mathrm{N}_{2}$, and shipped to the Cell Biology and Aging Section, San Francisco Veterans Administration Medical Center, for subsequent analysis. The tissues were maintained at $-70{ }^{\circ} \mathrm{C}$ until thawed and microsomes prepared (Schmucker and Wang, 1980a). NADPH cytochrome $\mathrm{P}-450$ reductase activity was estimated by measuring the rate of cytochrome $c$ reduction by intact microsomes and the values expressed as nmoles of cytochrome $c$ reduced per min per mg of microsomal protein (Masters et al., 1967). The microsomal concentration of this enzyme was measured using a rabbit anti-rat liver cytochrome $\mathrm{P}-450$ reductase polyclonal antibody in an ELISA. The total cytochromes P-450 content of the microsomes was estimated by (a) the CO-binding difference spectra (Omura and Sato, 1964) and (b) an ELISA using a rabbit anti-rat liver cytochromes P-450 polyclonal antibody. The relative microsomal concentration of the phenobarbital-inducible cytochromes $\mathrm{P}-450_{\mathrm{b}+\mathrm{e}}$ was determined in an ELISA using a murine monoclonal antibody directed against these rat isozymes (Bonkovsky et al., 1985).

Estimates of the membrane content of the male-specific cytochrome P-450, P-450 , were obtained via immunoblots. Essentially, microsomes were electrophoresed under denaturing conditions, transferred to nitrocellulose, blotted with a colloidal gold-labeled murine monoclonal antibody directed against rat liver cytochrome $\mathrm{P}-450_{\mathrm{h}}$, silver enhanced and subjected to densimetric analysis. The monoclonal antibody $\left(\mathrm{M}_{16}\right)$ was generously provided by Dr. Edward Morgan, Department of Pharmacology, Emory University, Atlanta, GA (Morgan et al., 1987). Protein was determined by the method of Lowry et al. (1951). The data from the various age groups and dietary regimens were compared and subjected to statistical analysis (Student's $t$-test). A linear regression analysis was performed on the data within the FF and $\mathrm{CR}$ groups in order to assess the effect of aging alone.

\section{RESULTS}

Full-fed (FF) rats steadily increased in body weight between 7 and 36 months of age (16\%), whereas the body weight of CR animals increased $14 \%$ through 18 months and subsequently declined to where the very old animals were slightly smaller than the corresponding young adults (Table 1). It should be noted that some of the very old CR animals were moribund, and much of the body weight loss occurred during the month prior to sacrifice. Liver weights exhibited a similar pattern with those of FF rats being significantly larger than those of CR animals. The liver weight/body weight ratios were identical regardless of age or feeding regimen $(2.5-3.2 \%)$. In general, the microsomal protein yields declined, but this was significant $(p<.01)$ only in the CR rats.

The specific activity of microsomal NADPH cytochrome $c(\mathrm{P}-450)$ reductase declined $38 \%(p<.01 ; r=.63)$ in FF rats during aging (Table 2 ). On the contrary, reductase activity in CR animals increased (23\%; $p=n$.s.) during the same age span, and the oldest animals (CR > 36) exhibited activities similar to those measured in the young $C R$ rats (CR7). The CR values (a) did not decline significantly during aging $(r=.04)$, (b) were significantly greater in mature rats vs similarly aged FF animals $(p<.01)$, and (c) in old and very old CR rats were not significantly different from those of young and mature FF animals. The microsomal content of immunoprecipitable $\mathrm{P}-450$ reductase correlated with measurements of enzyme activity, and the difference in reductase content between young and old FF microsomes was statistically significant $(p<.01 ; r=.40 ;$ Table 2$)$. The reductase content of $\mathrm{CR}$ microsomes increased twofold between maturity and old age $(p<.01)$ followed by a $43 \%$

Table 1. Effects of Age and Caloric Restriction on Body Weight, Liver Weight, and Yield of Hepatic Microsomal Protein in Male L-W Rats

\begin{tabular}{|c|c|c|c|c|c|c|c|}
\hline & \multicolumn{7}{|c|}{ Animal Age and Dietary Regimen } \\
\hline & FF7 & FF 18 & $\mathrm{FF}<36$ & CR7 & CR 18 & $\mathrm{CR}<36$ & $\mathrm{CR}>36$ \\
\hline Body weight (g) & $386 \pm 36^{a}$ & $450 \pm 26^{b}$ & $449 \pm 69^{c}$ & $261 \pm 39^{d}$ & $298 \pm 9 c$ & $281 \pm 41^{r}$ & $224 \pm 348$ \\
\hline Liver weight (g) & $10.7 \pm 1^{a}$ & $11.3 \pm 0.9^{b}$ & $12.7 \pm 2.4^{c}$ & $7.2 \pm 0.4^{d}$ & $8.4 \pm 0.3 e$ & $8.3 \pm 1.1^{r}$ & $7.1 \pm 1.78$ \\
\hline Microsomal protein $(\mathrm{mg} / \mathrm{g})$ & $13 \pm 5.3$ & $15.1 \pm 4.4$ & $9.6 \pm 2.9$ & $17 \pm 4.3^{\mathrm{a}}$ & $11.3 \pm 3.2^{\mathrm{b}}$ & $8 \pm 3.1^{c}$ & $11 \pm 4.7^{\mathrm{d}}$ \\
\hline
\end{tabular}

Notes. All values represent the means of 6-18 animals $\pm S D ; \mathrm{FF}=$ ad libitum fed; $\mathrm{CR}=30 \%$ caloric restricted.

Statistical analysis: Student's $t$-test; $p<.0$ I; Body Weight: a $>$ b,d,e,f,g; b $>$ e; c $>$ f,g; Liver Weight: a $>$ d,e,f,g; b $>$ e; $c>$ f,g; $d<$ e; Microsomal Protein: $a>b, c, d$.

Table 2. Effects of Age and Caloric Restriction on Activity and Concentration of Microsomal NADPH Cytochrome c (P-450) Reductase in Male L-W Rats

\begin{tabular}{|c|c|c|c|c|c|c|c|}
\hline & \multicolumn{7}{|c|}{ Animal Age and Dietary Regimen } \\
\hline & FF7 & FFI8 & $\mathrm{FF}<36$ & CR7 & CR18 & $\mathrm{CR}<36$ & $\mathrm{CR}>36$ \\
\hline Activity (nmol $\cdot \mathrm{min} \cdot \mathrm{mg}^{-1}$ ) & $82 \pm 17^{a}$ & $55 \pm 12^{b}$ & $51 \pm 13^{c}$ & $69 \pm 14$ & $79 \pm 8^{d}$ & $85 \pm 38$ & $68 \pm 31$ \\
\hline Concentration $\left(\right.$ Titer $\left.^{-1}\right)$ & $210 \pm 57^{a}$ & $81 \pm 52^{b}$ & $103 \pm 74^{c}$ & $144 \pm 61^{d}$ & $134 \pm 34$ & $345 \pm 163^{e}$ & $195 \pm 123^{f}$ \\
\hline
\end{tabular}

Notes. All values represent the means of 5-16 animals $\pm S D ; \mathrm{FF}=$ ad libitum fed; $\mathrm{CR}=30 \%$ caloric restricted; enzyme concentration measured in ELISA and expressed as the reciprocal titer.

Statistical analysis: Student's t-test; $p<.01$; Reductase Activity: a $>$ b,c; b $<$ d; Reductase Concentration: $\mathrm{a}>\mathrm{b}, \mathrm{c} ; \mathrm{c}<\mathrm{f} ; \mathrm{d}<\mathrm{e}$. 
decline $(r=.29)$. Still, the membranes of very old $C R$ animals ( $C R>36$ ) contained significantly more immunoprecipitable $\mathrm{P}-450$ reductase in comparison to the microsomes of young adult FF rats $(p<.01)$.

The cytochromes P-450 content of FF rat liver microsomes, measured by CO-binding Soret spectra, declined $55 \%$ between 7 and 36 months of age $(p<.01 ; r=.66$; Table 3 ). This value increased by $63 \%$ during the same age span in CR animals ( $p=$ n.s.) and subsequently declined such that the young adult values were significantly higher than those in the very old rats $(p<.01)$. As a result of these age-related shifts, there was no positive correlation between age and cytochromes $\mathrm{P}-450$ concentration $(r=.003)$. A less striking age-related decline occurred in the microsomal concentration of immunoprecipitable cytochromes P-450 in the ad libitum fed animals $(18 \% ; r=.09)$, whereas the concomitant increase and decrease in the CR rats correlated with that measured by CO-binding spectra $(p<.01)$ and precluded a positive correlation with increasing age $(r=$ .25; Table 3 ). The relative concentration of phenobarbitalinducible cytochrome P-450 isozymes, P-450 ${ }_{b+e}$, remained essentially unchanged and increased $(p=n$.s.) during aging in $\mathrm{FF}$ and $\mathrm{CR}$ rats, respectively (Table 3 ). Although the levels of cytochrome P-450 ${ }_{b+e}$ in old and very old CR rats were markedly higher than those measured in FF rats of any age, these differences were not statistically significant.

Qualitative assessment of immunoblots suggested that the relative concentration of male-specific cytochrome $\mathrm{P}-450$ $\left(\mathrm{P}-450_{\mathrm{h}} ; 16 \alpha\right.$-hydroxylase) declined substantially in both FF and $C R$ rats as a function of increasing age. Subsequent densimetric analysis confirmed that this sex-dependent P-450 isozyme declined $82 \%(p<.01 ; r=.96)$ and $65 \%(p$ $<.01 ; r=.92)$ between 7 and 30 months of age in FF and
CR rats, respectively (Table 4 ). Similar values were obtained regardless of whether microsomes from individual animals or pooled samples were blotted. Unlike the concentrations of total cytochromes P-450 or the isozymes P$450_{b+e}$, the microsomal content of $P-450_{h}$ in young CR rats was less than half that measured in similarly aged $\mathrm{FF}$ animals $(p<.01)$

\section{DisCUSSION}

The reduction in microsomal NADPH cytochrome $c$ (P-450) reductase activity during aging in the FF rats compares favorably with data from aging male rats of different strains (Schmucker and Wang, 1980a; 1980b; Kato and Takanaka, 1968; Gold and Widnell, 1974; Rikans and Notley, 1981; 1982). Furthermore, the increased values measured across the same age span in the CR rats agree favorably with the $20-50 \%$ increases in liver monooxygenase activities reported following (a) $50 \%$ food restriction for 7 weeks in post-weanling (Sachan, 1982; Sachan and Das, 1982) and (b) caloric restriction through presenescence in rats (Leakey et al., 1989). The report by Leakey et al. (1989) notwithstanding, the present study is the first to demonstrate that caloric restriction postpones the well-documented decline in the activity of this important hepatic microsomal enzyme in truly old rats. The slight decline in the microsomal content of immunoprecipitable reductase in FF animals corresponds with the concomitant loss of specific activity. Reduced enzymatic activity of microsomal and soluble P-450 reductase during aging in ad libitum fed male Fischer rats occurs concurrently with an accumulation of catalytically inactive, immunoprecipitable enzyme (Schmucker and Wang, 1983). In fact, the total liver complement of $\mathrm{P}-450$ reductase (active plus inactive) in old Fischer rats is considerably greater than

Table 3. Effects of Age and Caloric Restriction on Relative Microsomal Concentrations of the Total Cytochromes P-450 and the Isozymes P- $450_{b+c}$ in Male L-W Rats

\begin{tabular}{|c|c|c|c|c|c|c|c|}
\hline & \multicolumn{7}{|c|}{ Animal Age and Dietary Regimen } \\
\hline & FF7 & FFI8 & $\mathrm{FF}<36$ & CR7 & CR 18 & $\mathrm{CR}<36$ & $\mathrm{CR}>36$ \\
\hline Total P-450' (nmol/mg) & $0.47 \pm .1^{\mathrm{a}}$ & $0.35 \pm .1^{b}$ & $0.21 \pm .1^{\mathrm{c}}$ & $0.33 \pm . .^{\mathrm{d}}$ & $0.37 \pm .1$ & $0.54 \pm .4$ & $0.23 \pm .1^{e}$ \\
\hline Total P-4502 $\left(\right.$ Titer $\left.^{-1}\right)$ & $401 \pm 88^{a}$ & $273 \pm 179$ & $330 \pm 240^{\circ}$ & $405 \pm 255^{\mathfrak{c}}$ & $399 \pm 96^{d}$ & $992 \pm 465^{e}$ & $505 \pm 321^{i}$ \\
\hline P- $450_{b+c}{ }^{3}\left(\right.$ Titer $\left.^{-1}\right)$ & $257 \pm 85$ & $178 \pm 40$ & $222 \pm 145$ & $343 \pm 49$ & $230 \pm 98$ & $423 \pm 218$ & $433 \pm 169$ \\
\hline
\end{tabular}

Notes. All values represent the means of 5-16 animals $\pm S D ; \mathrm{FF}=$ ad libitum fed; $\mathrm{CR}=30 \%$ caloric restricted.

'Total cytochromes P-450 measured by CO-binding difference spectra.

${ }^{2}$ Total cytochromes P-450 measured by ELISA and expressed as the reciprocal titer.

${ }^{3}$ Cytochromes P-450 $0_{b+c}$ measured by ELISA and expressed as the reciprocal titer; no statistically significant differences between these values.

Statistical analysis: Student's $t$-test; $p<.0$ I; Total P-450 (CO-binding): a $>$ b,c,d,e; d $>$ e; Total P-450 (ELISA): a $<$ e; b $<$ e; c $<$ e; $d<$ e; e $>$ f.

Table 4. Effects of Age and Caloric Restriction on the Relative Microsomal Concentration of the Male Specific Cytochrome P-450 in Male L-W Rats

\begin{tabular}{lccccccc}
\hline \hline & \multicolumn{7}{c}{ Animal Age and Dietary Regimen } \\
\cline { 2 - 8 } & FF7 & FF18 & FF $<36$ & CR7 & CR18 & CR $<36$ & CR $>36$ \\
\hline P-450 $(\mathrm{Ab} / \mu$ g protein) & $2 \pm 0.2^{\mathrm{a}}$ & - & $0.37 \pm 29^{\mathrm{b}}$ & $0.80 \pm .23^{\mathrm{c}}$ & - & $0.28 \pm .1^{\mathrm{d}}$ & $0.03 \pm .03^{\mathrm{c}}$ \\
\hline
\end{tabular}

Notes. Data derived from densimetric scans of immunoblots and the values are expressed as the mean absorbance $\left(A_{540}\right)$ for $4-5$ animals per $\mu g$ of microsomal protein $\pm S D$.

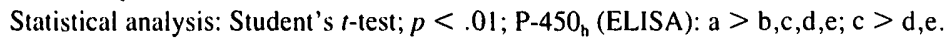


that of young adult animals (Schmucker et al., 1986). The difference between these data and those of the present study may reflect differences in animal strain, diet, and method of measurement, i.e., radial immunodiffusion vs ELISA or the fact that microsomal reductase concentration was not measured directly in the previous study. The absence of characterization studies precludes any conclusions concerning the nature and catalytic efficiency of the P-450 reductase in old and very old $C R$ animals. Nevertheless, the microsomal content of this enzyme in old CR rats is two- to threefold greater than that measured in any age group of FF rats.

The decline in the total cytochromes P-450 content of the microsomes during aging in FF rats concurs with data from a number of studies, including our own (Schmucker and Wang, 1980a; 1980b; see Schmucker, 1985 for a review). The observation that $\mathrm{CR}$ results in an increase in the total cytochromes P-450 content of male rat liver microsomes is not novel. Several laboratories have reported similar findings in young and presenescent (22 mos) rats (Sachan, 1982; Sachan and Das, 1982; Leakey et al., 1989). Most studies have estimated the cytochromes P-450 content by measuring the CO-binding difference spectra. However, we eliminated potential age-related alterations in the CO-binding capacity of the heme protein and substantiated our CO-binding data by measuring the relative concentrations of immunologically identifiable cytochromes P-450 using an ELISA. The good agreement between the results obtained with these two different methods supports our contention that CR (a) enhances the hepatic microsomal content of cytochromes P-450 in old rats, and (b) postpones the well-documented age-dependent decline in this parameter to later in the life span.

Leakey et al. (1989) recently reported that CR reduced or reversed many of the age-related declines in rat liver microsomal monooxygenases, but that the effects of this perturbation were not universal. For example, certain $\mathrm{N}$ demethylation and $\mathrm{O}$-deethylation activities were reduced in 22-month-old ad libitum fed and CR rats. However, the fact that these investigators failed to use truly old rats may confuse the interpretation of some of these data. They observed that the substrate-dependent activity for cytochromes $\mathrm{P}-450_{\mathrm{b}+\mathrm{e}}$, 7-pentoxyresorufin-O-dealkylase, declined significantly in 22-month-old ad libitum fed and CR animals vs 2month-old controls. Although our measurements of the relative microsomal concentrations of immunologically defined cytochromes $\mathrm{P}-450_{\mathrm{b}+\mathrm{e}}$ in animals up to 18 months of age do not refute their data, the twofold higher isozyme contents ( $p$ $=$ n.s.) in truly old and very old rats suggest that CR does, indeed, enhance the activities of these phenobarbital-inducible cytochrome P-450s during aging. It should be noted that the monooxygenase levels, especially the low microsomal concentrations of the phenobarbital-inducible cytochromes $\mathrm{P}-450_{b+e}$, in both studies reflect the facts that (a) phenobarbital-induced rats were not used, and (b) the noninduced levels of $\mathrm{P}-450_{\mathrm{b}+\mathrm{e}}$ are extremely low. This particular $\mathrm{P}-450$ isozyme was selected as a comparison to cytochrome $\mathrm{P}-450_{\mathrm{h}}$ because it is minimally responsive to shifts in the levels of serum testosterone. Furthermore, the present data are consistent with those demonstrating a marked age-related heterogeneity in the inducibility of hepatic cytochrome P-450 isozymes in male rats (Sun and Strobel, 1986).
Our measurements of immunologically defined malespecific cytochrome $\mathrm{P}-450_{\mathrm{h}}$ are in marked contrast to the relative activities of this monooxygenase reported by Leakey et al. (1989). The latter investigators reported $\mathrm{P}-450_{\mathrm{h}}$ activities (testosterone $2 \alpha$ and 16 $\alpha$-hydroxylase) (a) $88-98 \%$ reduced in 22-month-old vs 2-month-old ad libitum fed rats and (b) 4-14 fold greater in 22-month-old CR rats in comparison to similarly aged ad libitum fed animals. Our data show concomitant age-related declines in P-450 ${ }_{\mathrm{h}}$ concentration during aging in both ad libitum fed and CR rats. The absence of young CR animals in the study by Leakey et al. precludes any comparison with our results demonstrating a $\mathrm{CR}$-induced loss of $\mathrm{P}-450_{\mathrm{h}}$ even in young adult rats. A number of immunoblots from old CR rat microsomes were smeared in comparison to the distinct bands obtained with membranes from other groups. Since (a) there was no evidence of lower molecular weight proteolytic products, (b) there were no significant differences in P-450 reductase activity between samples with discrete and smeared immunoblots, (c) the densimetric analyses were normalized for band width, and (d) identical densimetric data were obtained using either individual or pooled membrane samples, we suggest that these estimates correctly reflect the fact that CR has essentially no effect on the age-related decline in liver cytochrome $\mathrm{P}-450_{\mathrm{h}}$ content in male rats. In fact, $\mathrm{CR}$ may even promote this demasculinization of the monooxygenase system in young adult animals.

The microsomal concentration of androgen-sensitive cytochrome $\mathrm{P}-450_{\mathrm{h}}$ may be influenced by testosterone levels, but the data concerning the effects of CR on serum testosterone levels during aging are controversial. Snyder et al. (1988) reported higher serum testosterone levels in the same CR L-W rats used in the present study, whereas Chatterjee et al. (1989) reported that CR failed to affect the plasma concentrations of this steroid, at least in animals up to 27 months of age. The former study revealed age-related declines in serum testosterone of $53 \%$ and $46 \%$ in FF and CR rats, respectively, between 7 and 24 months of age. The values measured in old CR rats were only slightly higher than those found in the oldest FF animals. Our data do not correlate with either of the above studies since higher or similar, rather than lower, cytochrome $\mathrm{P}-450_{\mathrm{h}}$ concentrations would be anticipated in $\mathrm{CR}$ vs FF rats. An alternative interpretation may be that hormonal regulation of $\mathrm{P}-450_{\mathrm{h}}$ expression is uncoupled during aging and that $\mathrm{CR}$ has relatively little influence at more finite levels of regulation, e.g., androgen sensitivity or post-receptor processing.

This study suggests that minimal CR $(30 \%)$ delays documented age-related declines in the activity and microsomal concentrations of certain hepatic monooxygenases that occur in ad libitum fed male rats. This observation is consistent with the effect of $40 \%$ CR on the synthesis of another hepatic protein, $\alpha_{24}$-globulin, at least in Fischer 344 rats (Richardson et al., 1987). We are aware that Koizumi et al (1987) did not detect any effect of CR on several liver microsomal monooxygenases during aging in female mice. However, other studies have been unable to document significant age-dependent declines in the hepatic monooxygenases of ad libitum fed mice (Stohs et al., 1980; Birnbaum, 1980). We are aware that fasting and protein malnutrition influence hepatic 
monooxygenases, but chronic caloric restriction is markedly different from brief underfeeding or starvation (Gil et al., 1988; see Yang and Yoo, 1988, for a review). In conclusion, the present study is the first to demonstrate that long-term caloric restriction may (a) postpone the age-related declines in several liver microsomal monooxygenases, and (b) increase their concentrations in old male rats. Although the changes in the cytochromes $\mathrm{P}-450_{b+c}$ are not statistically significant, the fact that the levels of these isozymes are considerably greater in old CR vs FF rats supports this contention. On the other hand, at least one monooxygenase, male specific cytochrome $\mathrm{P}-450_{\mathrm{h}}$, is significantly reduced during aging in both FF and CR rats. These differential effects of $C R$ on the age-related changes within a single enzyme system raise important questions regarding the universality of this modulator of the aging process. The hepatic monooxygenases offer an easily accessible and well-defined system in which to examine the mechanism(s) whereby $\mathrm{CR}$ perturbs their regulation during aging.

\section{ACKNOWLEDGMENTS}

Address correspondence to Dr. Douglas L. Schmucker, Cell Biology and Aging Section (151E), VA Medical Center, 4150 Clement Street, San Francisco, CA 94121 .

\section{REFERENCES}

Birnbaum, L. S. Altered hepatic drug metabolism in senescent mice. Exp. Gerontol. 15:259-267; 1980.

Bonkovsky, H. L.; Hauri, H. P.; Marti, U.; Gasser, R.; Meyer, U. A. Cytochrome P-450 of small intestinal epithelial cells. Gastroenterology $88: 458-467 ; 1985$.

Chatterjee, B.; Fernandes, G.; Yu, B. P.; Song, C.; Kim, J. M.; Demyan, W.: Roy, A. K. Calorie restriction delays age-dependent loss in androgen responsiveness of the rat liver. FASEB J. 3:169-173; 1989.

Gil, L.; Vasquez, H.; Orellana, M.; Selkirk, J.; Wold, F.; Strobel, H. W. Purification and characterization of liver cytochrome P-446 isolated from protein energy malnourished rats. Mol. Cell. Biochem. 79:5-16; 1988.

Gold, G.; Widnell, C. Response of NADPH cytochrome c reductase and cytochrome P-450 in hepatic microsomes to treatment with phenobarbital-differences in rat strains. Biochem. Pharmacol. 24:2105-2106; 1974.

Kamataki, T.; Maeda, K.; Shimada, M.; Kitani, K.; Nagai, T. Y.; Kato, R. Age-related alteration in the activities of drug-metabolizing enzymes and contents of sex-specific forms of cytochrome P-450 in liver microsomes from male and female rats. J. Pharmacol. Exp. Ther. 233:222$228 ; 1985$.

Kato, R.; Takanaka, A. Effect of phenobarbital on electron transport system, oxidation and reduction of drugs in liver microsomes of rats of different age. J. Biochem. (Tokyo) 63:406-408; 1968

Kitagawa, H.; Fujita, S.; Suzuki, T.; Kitani, K. Disappearance of sex difference in rat liver drug metabolism in old age. Biochem. Pharmacol. 34:579-581; 1985.

Koizumi, A.; Weindruch, R.; Walford, R. L. Influences of dietary restriction and age on liver enzyme activities and lipid peroxidation in mice. J. Nutr. 117:361-367; 1987.

Leakey, J. A.; Cunny, H. C.; Bazare, J., Jr.; Webb, P. J.; Feuers, R. J.; Duffy, P. H.; Hart, R. W. Effects of aging and caloric restriction on hepatic drug metabolizing enzymes in the Fischer 344 rat. I. The cytochrome P 450-dependent monooxygenase system. Mech. Ageing Dev. 48:145-155; 1989.

Lowry, O. H.; Rosebrough, N. J.; Farr, A. L.; Randall, R. J. Protein measurement with the Folin phenol reagent. J. Biol. Chem. 193:265$275 ; 1951$.

Masoro, E. Nutrition and aging - a current assessment. J. Nutr. 115:842$848 ; 1985$.
Masters, B. S.; Williams, C., Jr.; Kamin, H. The preparation and properties of TPNH cytochrome $\mathrm{c}$ reductase from pig liver. Methods Enzymol. 10:565-573; 1967

Morgan, E.; Ronnholm, M.; Ake-Gustafsson, J. Preparation and characterization of monoclonal antibodies recognizing unique epitopes on sexually differentiated rat liver cytochrome P-450 isozymes. Biochemistry 26:4193-4198; 1987

Omura, T.; Sato, R. The carbon monoxide-binding pigment of liver microsomes. II. Solubilization, purification and properties. J. Biol Chem. 239:2379-2385; 1964.

Richardson, A.; Butler, J. A.; Rutherford, M. S.; Semsei, I.; Gu, M. Z.; Fernandes, G.; Chiang, W. H. Effect of age and dietary restriction on the expression of $\alpha_{2 u}$-globulin. J. Biol. Chem. 262:12821-12825; 1987.

Rikans, L. E.; Notley, B. A. Substrate specificity of age-related changes in the inducibility of hepatic microsomal monooxygenases in middle-aged rats. Mech. Ageing Dev. 16:371-378; 1981.

Rikans, L. E.; Notley, B. A. Age-related changes in hepatic microsoma drug metabolism are substrate selective. J. Pharmacol. Exp. Ther 220:574-578; 1982.

Sachan, D. S. Modulation of drug metabolism by food restriction in male rats. Biochem. Biophys. Res. Commun. 104:984-988; 1982.

Sachan, D. S.; Das, S. K. Alterations of NADPH-generating and drugmetabolizing enzymes by feed restriction in male rats. J. Nutr. 112:2301-2308; 1982.

Schmucker, D. L. Age-related changes in drug disposition: an update Pharmacol. Rev. 37:133-148; 1985.

Schmucker, D. L.; Wang, R. K. Age-related changes in liver drugmetabolizing enzymes. Exp. Gerontol. 15:321-329; 1980a.

Schmucker, D. L.; Wang R. K. Age-related changes in liver drug metabolism: structure vs function. Proc. Soc. Exp. Biol. Med. 165:178-197 1980b.

Schmucker, D. L.; Wang, R. K. Effects of aging and phenobarbital on the rat liver microsomal drug-metabolizing system. Mech. Ageing Dev. 15:189-202; 1981

Schmucker, D. L.; Wang, R. K. Age-dependent alterations in rat liver microsomal NADPH cytochrome $\mathrm{c}(\mathrm{P}-450)$ reductase: a qualitative and quantitative assessment. Mech. Ageing Dev. 21:137-156; 1983.

Schmucker, D. L.; Vessey, D. A.; Wang, R. K.; Maloney, A. G. Aging, sex and the liver microsomal drug-metabolizing system in rodents and primates. In: Kitani, K., ed. Liver and aging - 1986. Amsterdam Elsevier-North Holland, 1986:3-14.

Snyder, D. L., ed. Dietary restriction and aging: progress in clinical and biological research, Vol. 287. New York: Alan R Liss, 1989.

Snyder, D. L.; Wostmann, B. S. Growth rate of male germfree Wistar rats fed ad libitum or restricted natural ingredient diet. Lab. Animal Sci. 37:320-325; 1987

Snyder, D. L.; Wostmann, B. S.; Pollard, M. Serum hormones in dietrestricted gnotobiotic and conventional Lobund-Wistar rats. J. Gerontol. Biol. Sci. 43:B 168-B 173; 1988.

Snyder, D. L.; Pollard, M; Wostmann, B. S.; Luckert, P. Life span, morphology, and pathology of diet-restricted germ-free and conventional Lobund-Wistar rats. J. Gerontol. Biol. Sci. 45:B52-B58; 1990.

Stohs, S. J.; Al-Turk, W. A.; Hassing, J. M. Altered drug metabolism in hepatic and extrahepatic tissues in mice as a function of age. Age 3:88$92 ; 1980$.

Sun, J. ; Strobel, H. W. Aging affects the drug metabolism systems of rat liver, kidney, colon and lung in a differential fashion. Exp. Gerontol. 21:523-534; 1986.

Walford, R. L.; Harris, S. B.; Weindruch, R. Dietary restriction and aging: historical phases, mechanisms, current directions. J. Nutr. 117:1650 1654; 1987.

Wu, B.; Conrad, C. C.; Richardson, A. Effect of aging and dietary restriction on the expression of $\alpha_{2 u}$-globulin in two strains of rats. In Snyder, D. L., ed. Dietary restriction and aging. New York: Alan R. Liss, 1989:301-309.

Yang, C. S.; Yoo, J. H. Dietary effects on drug metabolism by the mixedfunction oxidase system. Pharmacol. Ther. 38:53-72; 1988. 